\title{
Role of Zip1 in the regulation of NPY expression by MLT to promote fracture healing in rats
}

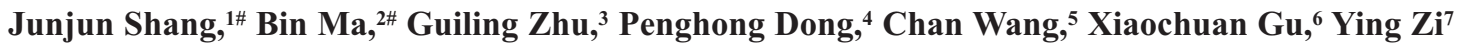 \\ ${ }^{1}$ Graduate Training Base of Jinzhou Medical University, Air Force Hospital of the Northern Theater of Chinese \\ People's Liberation Army (PLA), Shenyang, Liaoning \\ ${ }^{2}$ Division of Spine Surgery, Department of Orthopaedics, Tongji Hospital, Tongji University School of Medicine, \\ Shanghai \\ ${ }^{3}$ Department of Emergency Medicine, Zoucheng People's Hospital, Jining \\ ${ }^{4}$ Department of Critical Medicine, 242 Hospital, Shenyang, Liaoning \\ 5Shenyang Children's Hospital, Shenyang, Liaoning \\ ${ }^{6}$ Department of Orthopedics, Changhai Hospital, Navy Medical University, Shanghai \\ ${ }^{7}$ Department of Emergency Medicine, Graduate Training Base of Jinzhou Medical University, Air Force Hospital of \\ the Northern Theater of Chinese People's Liberation Army (PLA), Shenyang, Liaoning, China \\ \#These authors contributed equally
}

\begin{abstract}
Our previous study documented that melatonin (MLT) induced the osteogenic differentiation of mesenchymal stem cells (MSCs) and promoted the healing of femoral fractures in rats via the neuropeptide Y (NPY)/neuropeptide Y1 receptor (NPY1R) signaling pathway. MLT treatment upregulated the expression of the zinc uptake transporter zinc transporter 1 (Zip1) in nerve cells. Prior research demonstrated that oral zinc upregulated NPY expression. MSCs were isolated from rat bone marrow and identified using flow cytometry in our study. The results showed that MLT treatment upregulated NPY and NPY1R levels in MSCs with osteogenic differentiation, which was accompanied by upregulated Zip1 expression. However, the MLT-induced osteogenic differentiation of MSCs was reversed after interference of Zip1 expression. It was confirmed by the decreased alkaline phosphatase (ALP) level; downregulated activities of type I collagen $\alpha 1$ chain (COL1A1), osteocalcin (OCN), runt-related transcription factor 2 (Runx2) and ALP; and reduced mineralized nodule formation. MLT promoted fracture healing in rats with femoral fracture, which was accompanied by increased expression of NPY and NPY1R and significantly increased expression of Zip1. In contrast, the silencing of Zip1 expression reversed MLT-mediated fracture healing. In summary, Zip1 participated in the regulation of the NPY/NPY1R signaling pathway via MLT to promote the osteogenic differentiation of MSCs and fracture healing.
\end{abstract}

Key words: Zip1 protein; lentivirus; melatonin; neuropeptide Y; neuropeptide Y receptor Y1; mesenchymal stem cells; fracture healing.

Correspondence: Dr. Ying Zi, Department of Emergency Medicine, Graduate Training Base of Jinzhou Medical University, Air Force hospital of the northern theater of Chinese PLA, Shenyang, Liaoning,110042, China. E-mail:m18909827859@163.com

Contributions: JS, BM, carried out the studies, participated in collecting the data, drafted the manuscript and were responsible and accountable for the accuracy and integrity of the work; XG, YZ, performed the statistical analysis and participated in the study design; GZ, PD, CW, participated in the acquisition, analysis, or interpretation of the data and drafted the manuscript. All authors have read and approved the final manuscript.

Conflict of interest: The authors declare that they have no competing interests, and all authors confirm accuracy.

Funding: The study received financial support from the Science and Technology Plan of Shenyang (18-014-4-48); Shanghai Natural Science Foundation General Project (No. 17ZR1422900); and Changhai Hospital Youth Start-up Fund (No. CH201803).

Availability of data and materials: The data used to support the findings of this study are available from the corresponding author upon request.

Ethical Approval: The study was approved by the Institutional Ethics Committee of Changhai Hospital, Second Military Medical University, Shanghai, China. 


\section{Introduction}

There has been a remarkable increase in the incidence of trauma-induced fracture with the rapid development of society. Because of the influence of age and disease factors, patients frequently suffer difficulty in healing and subsequent complications, such as limb disuse atrophy. Approximately 5\% to $10 \%$ of all fractures cannot be completely healed, which imposes a heavy physiological and economic burden on individuals. ${ }^{1}$ Therefore, it is of great economic and social significance to study the mechanism of fracture healing in clinical practice and experimental technology and examine the key targets and effective therapeutic approaches to promote fracture healing.

Zinc is an essential trace element for human growth. It is widely recognized that zinc plays a role in bone formation, bone reconstruction and fracture healing. Recent studies showed that zinc levels affected spinal fusion in rats. ${ }^{2}$ Zinc transporter 1 (Zip1) is a major zinc uptake transporter that is intimately correlated with the accumulation of zinc in vivo. ${ }^{3,4}$ The overexpression of Zip1 induced osteogenic differentiation in mesenchymal stem cells (MSCs). ${ }^{5}$ Bone morphogenetic protein 2 (BMP2) may regulate the osteogenic differentiation of MSCs via Zip1 upregulation. ${ }^{6}$ Therefore, Zip1 may be involved in fracture healing by affecting the osteogenic differentiation of MSCs, but its mechanism is not clear. Our previous study found that melatonin (MLT) treatment upregulated the expression of Zip1 in N2a cells under hypoxia treatment, ${ }^{7}$ which suggests a regulatory role of MLT in Zip1 expression. Prior research ${ }^{8}$ demonstrated that oral zinc upregulated neuropeptide Y (NPY) expression, and our study hypothesized that MLT would affect the level of NPY and its receptor via the upregulation of Zip1 expression and the osteogenic differentiation of MSCs. However, the role of Zip1 in the regulation of MLT on NPY level is not known. Our research group previously demonstrated increased NPY and NPY1R expression in the healing bone tissue and muscles around a fracture after MLT treatment in a rat model of femoral fracture, and an NPY1R inhibitor (BIBP3226) reversed the role of MLT in accelerating fracture healing. ${ }^{9}$. The present study isolated MSCs from rat bone marrow and measured the levels of Zip1, NPY and NPY1R during osteogenic differentiation. The role of Zip1 in MLT regulation of NPY and NPY1R expression was studied in the process of osteogenic differentiation of MSCs and the rat model of femoral fracture.

\section{Materials and Methods}

\section{Animals and reagents}

Male Sprague-Dawley rats were purchased from Liaoning Changsheng Technology Co., Ltd., China. All animals were housed in a room with an alternating 12-hour dark-light cycle with free access to food and water. The Second Military Medical University approved the study, which was completed in accordance with ethical guidelines stated in the Guide for the Care and Use of Laboratory Animals. MLT was obtained from SeraCare Life Sciences Inc, Shanghai, China (BBI solutions). BIBP3226 was purchased from Tocris, Shanghai, China. MLT was dissolved in or $1 \%$ alcohol, and BIBP3226 was dissolved in normal saline (NS).

\section{Construction and identification of Zip1 interfering lentivirus vector}

Lentivirus vectors interfering with Zip1 and its negative control were constructed according to the sequence of the Zip1 gene in rats (accession No. NM_001134577.1). After 72 h, Zip1 expression was identified using real-time quantitative PCR and Western blotting.

\section{Isolation and identification of MSCs}

MSCs were isolated from the bone marrow of 4-week-old rats. The experimental rats were euthanized via an intraperitoneal injection of sodium pentobarbital, and the femur and tibia were separated under sterile conditions. ${ }^{10}$ The marrow cavity was washed repeatedly with Dulbecco's Modified Eagle's Medium (DMEM; GIBCO, Grand Island, NY, USA). The cell suspension was centrifuged at $350 \mathrm{~g}$ for $10 \mathrm{~min}$, and the supernatant was discarded. The precipitate was mixed with erythrolysis buffer (Solarbio, Beijing, China) for $3 \mathrm{~min}$. Cells were subjected to centrifugation and washing, followed by culture in DMEM supplemented with $10 \%$ fetal bovine serum.

The specific antibody markers of MSCs were detected using flow cytometry. Positive antibody markers included CD90-FITC (eBioscience, San Diego, CA, USA) and CD105-FITC (Abcam, Cambridge, UK). Negative markers were CD34 (Abcam) combined with goat anti-rabbit IgG (Abcam) labeled with phycoerythrin and FITC-CD45. Cells stained with an isotype control (IgGFITC) were used as the negative control.

\section{Transfection of MSCs with Zip1 interfering lentivirus vector and culture}

MSCs at P5 and the logarithmic growth phase in good condition were digested with trypsin, followed by the adjustment of cell concentration to $1 \times 10^{5}$ cells $/ 9.6 \mathrm{~cm}^{2}$ and inoculation into a 6 -well plate. ${ }^{11}$ When the cells reached $30 \%-50 \%$ cell fusion, Zip1 interfering lentivirus vector was used to interfere with the cells. The cells were supplemented with $10 \mathrm{mg} / \mathrm{L}$ Polybrene and incubated overnight in a $5 \% \mathrm{CO}_{2}$ incubator at $37^{\circ} \mathrm{C}$. Cells were selected for subsequent experiments after drug addition and screening.

Four groups were established in this experiment: a control group, an MLT group, and two groups of MSCs transfected with Zip1 interfering lentivirus vector. Cells were cultured in osteogenic induction medium (OIM) containing DMEM, 10\% fetal rat serum, $100 \mathrm{nmol} / \mathrm{L}$ dexamethasone, $50 \mu \mathrm{mol} / \mathrm{L}$ ascorbic acid and 10 $\mathrm{mmol} / \mathrm{L}$ sodium $\beta$-glycerophosphate. No treatment was applied to cells in the control group, and cells in the MLT group received 2 $\mathrm{mmol} / \mathrm{L}$ MLT. The other two groups received $2 \mathrm{mmol} / \mathrm{L}$ MLT and $2 \mathrm{mmol} / \mathrm{L} \mathrm{MLT}+0.1 \mathrm{nmol} / \mathrm{L}$ BIBP3226 to study the role of Zip1 in MLT regulation of NPY in the process of the osteogenic differentiation of MSCs.

\section{Western blotting}

Total protein was extracted from bone using RIPA buffer (Beyotime Institute of Biotechnology, Haimen, China) containing $1 \%$ phenylmethylsulphonyl fluoride. A bicinchoninic acid (BCA) assay kit (Beyotime Institute of Biotechnology) was used to determine the protein concentration. The sample was resolved using $10 \%$ SDS-PAGE, transferred to a polyvinylidene difluoride membrane, and blocked with 5\% skim milk. The membrane was incubated with primary antibodies against Zip1 (1: 100; Santa Cruz Biotechnology, Santa Cruz, California, USA), NPY (1:100; Santa Cruz Biotechnology, Santa Cruz, CA, USA), NPY1R (1:500; Abcam), type I collagen $\alpha 1$ chain (COL1A1; 1:300; CST, Boston, MA, USA), runt-related transcription factor 2 (Runx2; 1:1000; Bioss, Beijing, China) and GAPDH (1:1000; Key-GEN, Nanjing, China) overnight at $4^{\circ} \mathrm{C}$. Membranes were incubated with horseradish peroxidase (HRP)-labeled secondary antibodies, and the proteins were visualized using enhanced chemiluminescence (Beyotime Institute of Biotechnology). The expression of target genes was evaluated using gray value analysis, which was normalized to the GAPDH reference gene. 


\section{Real-time PCR}

Total RNAs were separated from cells, and bones using TRIpure reagent (BioTeke, Beijing, China) according to the manufacturer's instructions. cDNA was obtained via reverse transcription with a super M-MLV reverse transcriptase (BioTeke). The following primers were used for real-time PCR:

ALP forward, 5'-CATCGGACCCTGCCTTAC-3' reverse, 5'- GGAGACGCCCATACCATC -3'; COL1A1 forward, 5'- CGATTCACCTACAGCACGCTTG -3' reverse, 5'- TGGGATGGAGGGAGTTTACACG -3'; OCN forward, 5'- TAAGGTGGTGAATAGACTCCG-3' reverse, 5'- TAAACGGTGGTGCCATAGAT-3'; Runx2 forward, 5'-CCATAACGGTCTTCACAAATC -3' reverse, 5'- GAGGCGGTCAGAGAACAAACT-3'; Zip1 forward, 5'-GAGCAGTCCAGTCCACCACA-3' reverse, 5'-GACCAGTACACAGGCACGCA-3'; NPY forward, 5'- CTATCCCTGCTCGTGTGTTT-3' reverse, 5'- TGAGATTGATGTAGTGTCGC-3'; NPY1R forward, 5'- GATGGACAAAATCAGGGACAG -3' reverse, 5'- AGAGCAGGAACAGCAGATTG -3'; GAPDH forward,5'- CGGCAAGTTCAACGGCACAG -3' reverse, 5'- CGCCAGTAGACTCCACGACAT-3';

The relative gene expression was calculated according to the $2^{-\triangle \Delta C T}$ method.

\section{Alizarin red staining}

Mineralized nodule formation was observed using alizarin red staining. MSCs transfected with control lentivirus or Zip1 interfering lentivirus vector were inoculated in 12-well plates, cultured in OIM, and treated with DMSO, MLT or MLT + BIBP3226. After 21 d of culture, cells were fixed with $4 \%$ paraformaldehyde for 30 min, followed by the addition of alizarin red staining solution at room temperature for $10 \mathrm{~min}$. Photography was performed using a Motic microscope $(100 \times)$. The absorbance of the release of alizarin red was determined using an automatic quantitative microplate reader (BioTek) at the wavelength of $570 \mathrm{~nm}$.

\section{Detection of alkaline phosphatase}

The activity of alkaline phosphatase (ALP) in cell supernatants was determined using a commercial kit (Nanjing Jiancheng Bioengineering Institute, China) according to the manufacturer's instructions.

\section{Establishment of femoral fracture model in rats}

Thirty 12-week-old rats were randomly divided into five groups: the model group, MLT group, MLT+Zip1 interference group, MLT+BIBP3226+Zip1 interference group, and control group, with 6 rats in each group. The femoral fracture model in rats was established according to previous research. ${ }^{12,13}$ Briefly, rats were anesthetized via an intraperitoneal injection of sodium pentobarbital, and the right femoral shaft was exposed via lateral incision. A transverse osteotomy was made with a needle file in the proximal-middle segment of the femur, followed by wound suture layer by layer. Rats in the MLT group received an intravenous injection of MLT solution at a dose of $30 \mathrm{mg} / \mathrm{kg}$ postoperatively. Rats in the MLT + Zip1 interference group received a postoperative intravenous injection of $30 \mathrm{mg} / \mathrm{kg} /$ day MLT, followed by a single injection of $100 \mu \mathrm{L} 3 \times 10^{7}$ of virus and matrix glue mixture at the fracture site on the $4^{\text {th }}$ day after surgery. ${ }^{14}$ Rats in the MLT+BIBP3226+Zip1 interference group were injected intravenously with $20 \mu \mathrm{g} / \mathrm{rat} / 2$ days BIBP3226 and $30 \mathrm{mg} / \mathrm{kg} /$ day MLT postoperatively and received a single injection of $100 \mu \mathrm{L} 3 \times 10^{7}$ of virus and matrix glue mixture at the fracture site on the $4^{\text {th }}$ day after surgery. Rats in the NC group had no treatment for the fracture. Rats were euthanized 8 weeks after treatment. The bone tissue samples of the injection site were extracted and fixed with a $10 \%$ colorless formaldehyde solution. The healing tissue at the fracture site was immediately frozen in liquid nitrogen in a $-80^{\circ} \mathrm{C}$ freezer for subsequent use.

\section{Histological staining}

The fixed specimens were embedded in paraffin and cut into serial sections at a thickness of $5 \mu \mathrm{m}$. The sections were evaluated using hematoxylin-eosin (H\&E) and Masson staining. The histological staining was evaluated using a commercial kit (SigmaAldrich, St. Louis, MO, USA). Photography was performed using an Olympus microscope $(40 \times$ and $100 \times)$.

\section{Statistical analysis}

Each experiment was repeated three times, and the results are expressed as the means $\pm \mathrm{SD}$. One-way or two-way analysis of variance was used for statistical analysis, followed by post-hoc analysis using GraphPad Prism 7 software. $\mathrm{p}<0.05$ indicated that the difference was statistically significant. There was no indication in the assessment of the normal distribution of data to justify the use of conventional ANOVA.

Table 1. General observation results of each group.

\begin{tabular}{lcccc} 
No. & Mental state & Temperature & Diet and drinking & Response to external stimuli \\
Blank control group & Good & Normal & Normal & Responsive \\
Model group & Obvious mental fatigue & Increase & Obvious decrease & Obviously slow response \\
\hline MLT group & Passable & Basically normal & Basically normal & Faster \\
MLT+Zipl interference group & Mild mental fatigue & Slight increase & Slight decrease & Slightly slow response \\
\hline MLT+BIBP3226+Zipl interference group & Mental fatigue & Increase & Obvious decrease & Slow response \\
\hline
\end{tabular}




\section{Results}

\section{Identification of Zip1 interfering lentivirus vector}

Three days after transfection, Western blotting and real-time PCR showed that the inhibition rate using Zip1 interfering lentivirus vector exceeded $70 \%$ (Figure $1 \mathrm{a}, \mathrm{b}$ ).

\section{Identification of MSCs}

MSCs were isolated from rat bone marrow, and the cell phenotype was evaluated using flow cytometry. As shown in Figure 2, the cells were CD34 and CD45 negative and CD90 and CD105 positive, which is consistent with the reported phenotype of MSCs.

\section{Role of Zip1 in MLT regulation of NPY and NPY1R in the promotion of the osteogenic differentiation of MSC}

The role of Zip1 in MLT regulation of NPY and NPY1R in the promotion of osteogenic differentiation of MSCs was investigated in vitro. DMSO, $2 \mathrm{mmol} / \mathrm{LMLT}$ or $2 \mathrm{mmol} / \mathrm{L} \mathrm{MLT}+0.1 \mathrm{nmol} / \mathrm{L}$ BIBP3226 were used to treat the cells in osteogenic differentiation medium, and cells were transfected with control lentivirus or Zip1 interfering lentivirus vector.

The results of Western blotting showed that MLT upregulated the protein levels of Zip1, NPY, NPY1R, COL1A1 and Runx2 in MSCs, and interference with the expression of Zip1 partially inhib- ited these effects. The inhibitory effect was more obvious with interference of the expression of Zip1 and NPY (Figure 3a). Realtime PCR results also detected that interference with Zip1 expression inhibited the MLT-mediated increase in ALP, COL1A1, OCN, and Runx 2 mRNA, and interfering with Zip1 and NPY expression increased the inhibitory effect (Figure 3b). Real-time PCR results also detected that interference with Zip1 expression inhibited the MLT-mediated increase in ALP, COL1A1, OCN, and Runx2 mRNA, and interference with Zip1 and NPY expression increased the inhibitory effect (Figure 3c). Alizarin red staining results indicated that MLT accelerated the osteogenic differentiation of MSCs, which was inhibited by interfering with the expression of Zip1. Suppression of the expression of Zip1 and NPY further inhibited the osteogenic differentiation of MSCs (Figure 3d). Role of Zip1 in the regulation of NPY and NPY1R by MLT to promote fracture healing in a rat model. A rat model of femoral fracture was established for further examination. General observation results are shown in Table 1. Western blotting analysis showed that the levels of Zip1, NPY, and NPY1R in bones increased during MLT treatment, and the function of interfering with Zip1 expression was inhibited. Interfering with the expression of Zip1 and NPY increased the inhibitory effect (Figure 4a). Real-time PCR analysis also demonstrated that the levels of Zip1, NPY and NPY1R in bones increased during MLT treatment, and the function of interfering with Zip1 expression was inhibited. Interfering with the
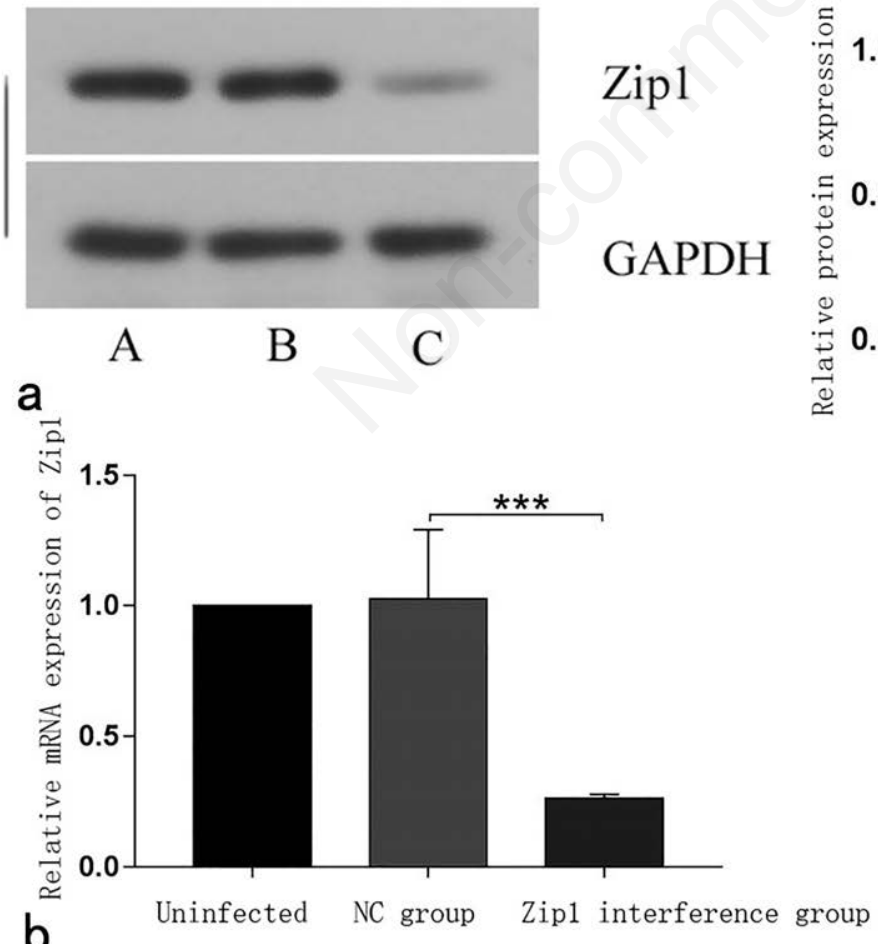

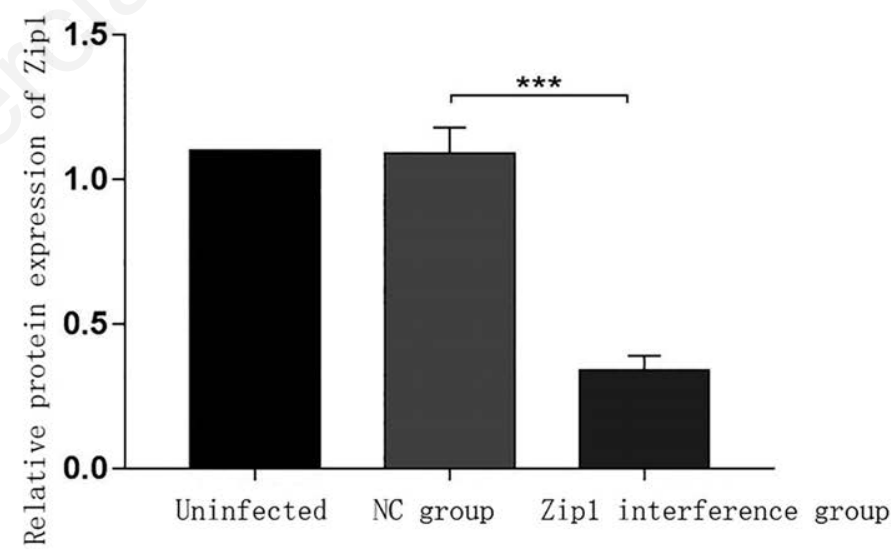

\author{
- group A (Uninfected group) \\ $\square$ group B (NC group) \\ group C (Zip1 interference group)
}

Figure 1. a) Zip1 expression level 3 days after transfection using Western blotting; b) Zip1 expression level 3 days after transfection using real-time PCR. 
expression of Zip1 and NPY increased the inhibitory effect (Figure 4b). H\&E staining results showed that the blank control group had normal bone morphology and obvious bone marrow cavity structure. The model group showed large-area granulation tissue hyperplasia and filling, a small amount of inflammatory cell infiltration, and a large number of osteoblasts. The basic bone structure was in the MLT group, and granulation tissue is formed in the local area. Compared with the model group, the number of bone cells increased, and more mature bone trabeculae were observed. Compared with the MLT group, the number of osteoclasts in the MLT+Zip1 interference group increased, and the bone tissue area decreased. The MLT+BIBP3226+Zip1 interference group had granulation tissue formation, but it was less than the model group (Figure 5a).

Masson staining results showed that there was no obvious collagen fiber formation in the blank control group. The model group showed a large area of collagen fibers, a large amount of hyperemia in the granulation formation, and osteoblasts in some areas. The formation of collagen fibers in the MLT group was less than the model group. The tissue structure showed that the collagen fiber area of the MLT+Zip1 interference group was higher than the MLT group. The MLT+BIBP3226+Zip1 interference group had a large number of osteoclasts, and the collagen area gradually increased (Figure 5b). The results of fracture healing area showed that MLT promoted fracture healing, and interference with Zip1 expression inhibited the effect of MLT on promoting fracture healing. Interfering with the expression of Zip1 and NPY further reduced the fracture healing area (Figure $5 \mathrm{c}$ ).

\section{Discussion}

The present study demonstrated the role of Zip1 in the regulation of the NPY/NPY1R signaling pathway by MLT to promote fracture healing. Our results suggested that MLT accelerated the osteogenic differentiation of MSCs and increased the levels of Zip1, NPY and NPY1R. In contrast, inhibition of Zip1 expression reversed MLT-mediated osteogenic differentiation, which was accompanied by decreased expression of NPY and NPY1R. MLT treatment in the established rat model of femoral fracture increased the expression of NPY, NPY1R and Zip1 in the callus. The inhibition of Zip1 expression reversed the role of MLT in promoting the expression of NPY and NPY1R in the callus and inhibited fracture healing. These results suggest that Zip1 mediates MLT regulation of NPY and NPY1R to promote fracture healing. The simultaneous inhibition of the expression of Zip1 and NPY further inhibited the osteogenic differentiation of MSCs and fracture healing, and MLT may play a role via receptor-dependent and -independent mechanisms. ${ }^{15,16}$ MLT may regulate NPY and NPY1R expression via the MLT receptor. Viruses are potent pathogens that have highly evolved to effectively deliver genetic material to susceptible host cells. The virus is incorporated into the host cell genome and produces relatively long-term expression of genetic information, or it exists in a free state outside the genome and avoids the risk of insertional mutagenesis. ${ }^{17,18}$ Adenovirus vector has been used in a preclinical bone repair model for a long time, and it exhibits good transduction efficiency. ${ }^{19,20}$ However, the immune response produced certain risks in an in vivo experiment. ${ }^{21}$ A lentivirus vector enables long-term protein expression with minimal immunogenicity, ${ }^{25,26}$ and it is commonly used in research. ${ }^{12,22-24}$ Therefore, the current experiment used lentivirus to interfere with the expression of Zip1. Fracture healing is a complex process in which the integration of cells, growth factors and extracellular matrix play crucial roles. ${ }^{27}$ Thirty-six hours after fracture, periosteal cells proliferate in the injured area that is adjacent to the fracture site. ${ }^{28}$ These cells may be susceptible to retrovirus infection, and a good result was obtained after the injection of a virus vector directly into the fracture site. ${ }^{14,29}$ Therefore, direct injection of a virus vector may be an effective method.
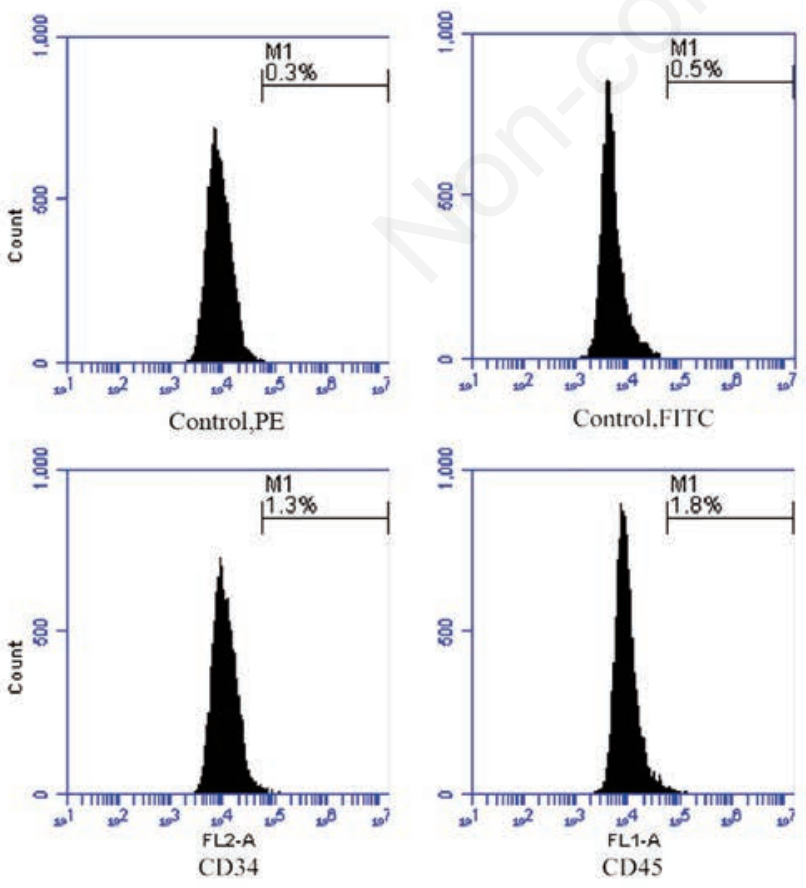
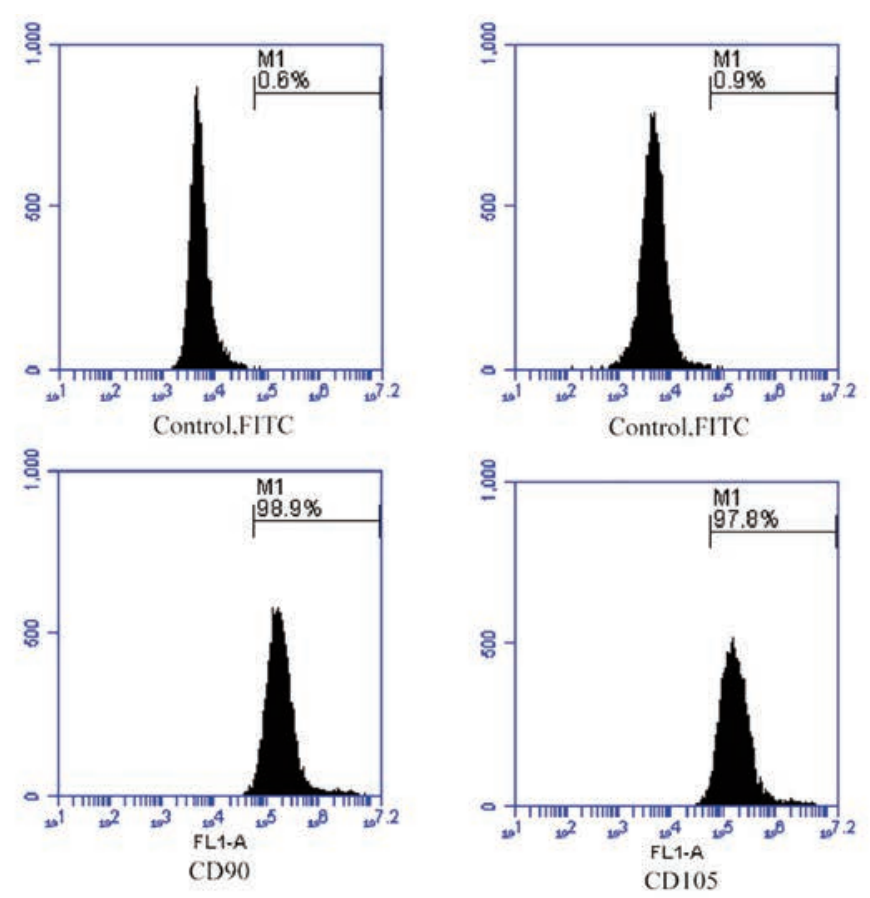

Figure 2. Percentages of CD34+, CD45+, CD90+ and CD105+ cells in MSCs using flow cytometry. 


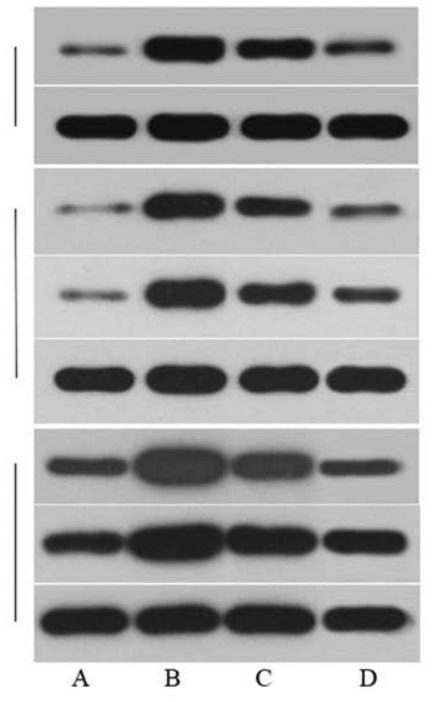

a

\section{Zip1 \\ GAPDH}

NPY

NPY1R

GAPDH

COL1A1

Runx2

GAPDH
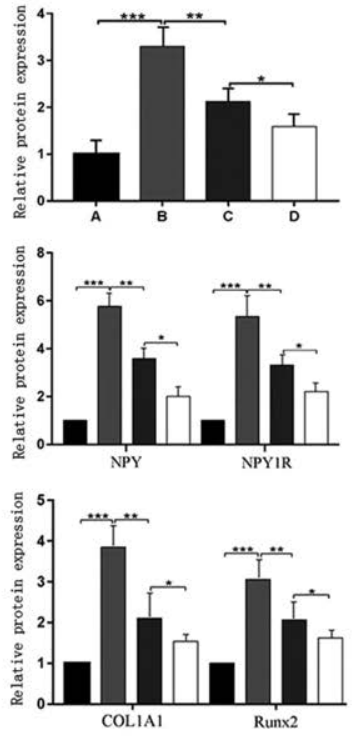

b
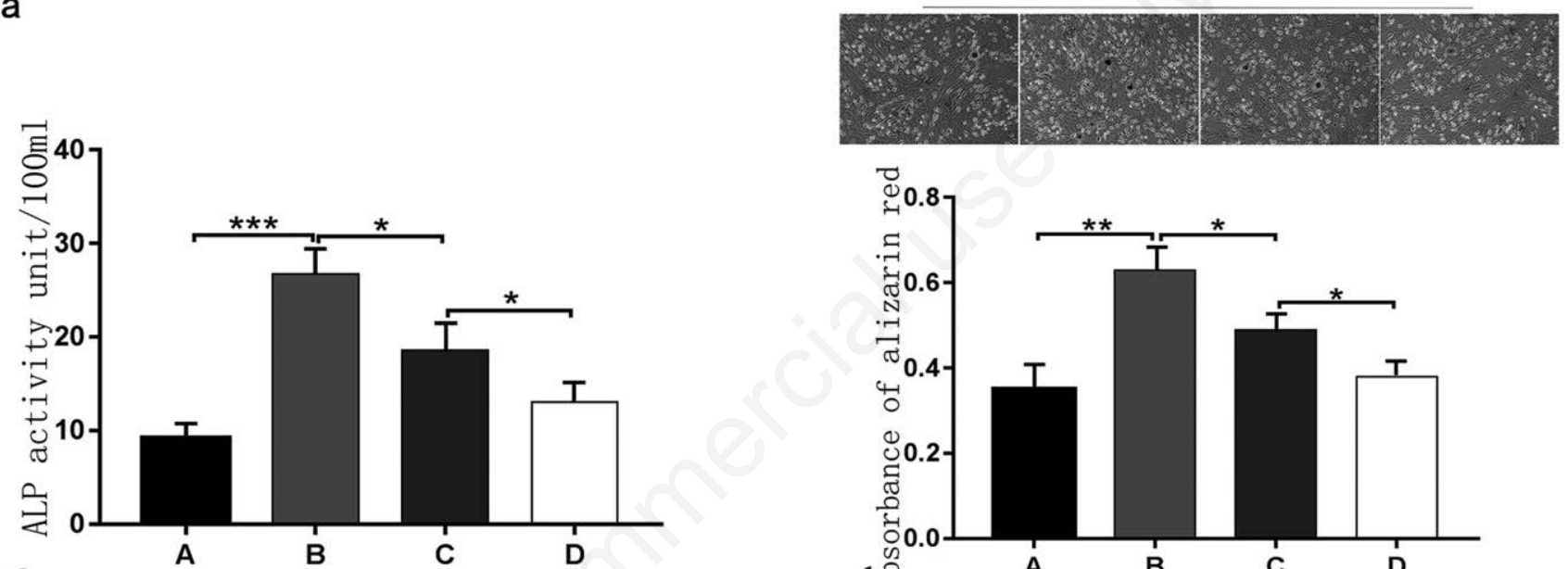

c

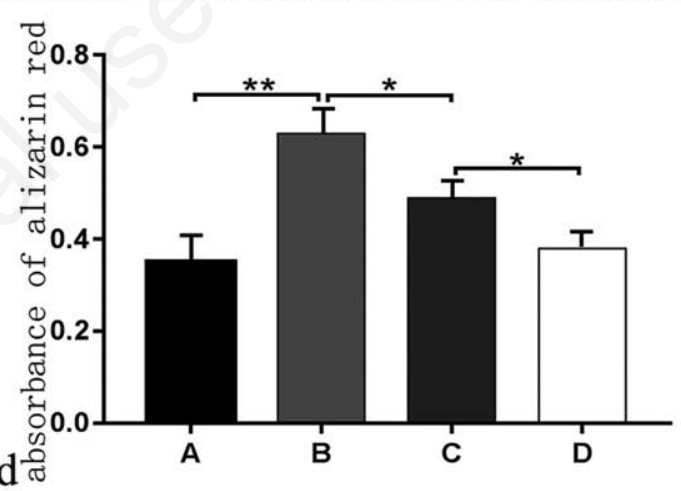

Figure 3. Induction of osteogenic differentiation from bone marrow MSCs and treatment with DMSO, $2 \mathrm{mmol} / \mathrm{LMLT}$ or $2 \mathrm{mmol} / \mathrm{L}$ MLT $+0.1 \mathrm{nmol} / \mathrm{L}$ BIBP3226. a) COL1A1 and Runx2 levels on the $7^{\text {th }}$ day, and Zip1, NPY and NPY1R expression levels using Western blotting on the $21^{\text {st }}$ day. b) ALP, COL1A1, OCN and Runx2 expression levels using real-time PCR on the $7^{\text {th }}$ day after treatment. c) The activity of ALP in supernatant using a commercial kit on the $21^{\text {st }}$ day. $d$ ) The osteogenic differentiation of MSCs using alizarin red staining on the $21^{\text {st }}$ day. The data are shown as mean \pm SD. ALP, alkaline phosphatase; COL1A1, collagen type I $\alpha 1$ chain; MLT, melatonin; BIBP3226, NPY1R inhibitor; OCN, osteocalcin; Runx2, runt-related transcription factor $2 ;{ }^{*} \mathrm{p}<0.05 ;{ }^{* *} \mathrm{p}<0.01 ;{ }^{* * *} \mathrm{p}<0.001$. Control groups: A and B; B and C; C and D. 


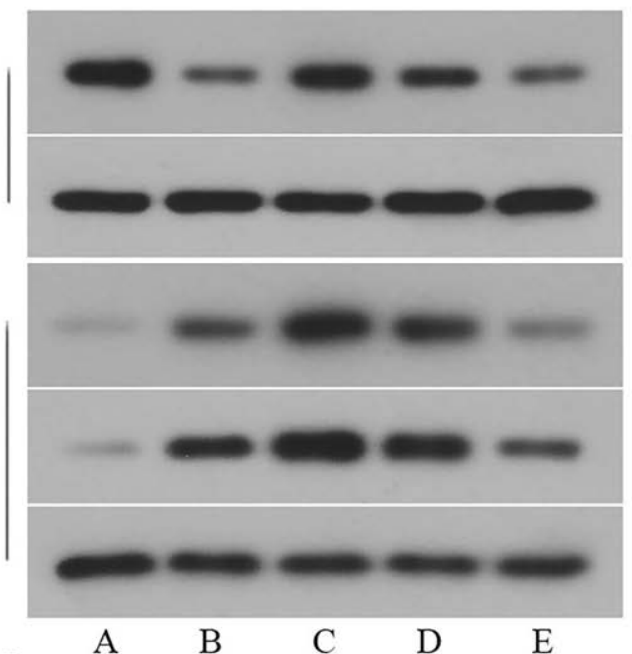

Zip1

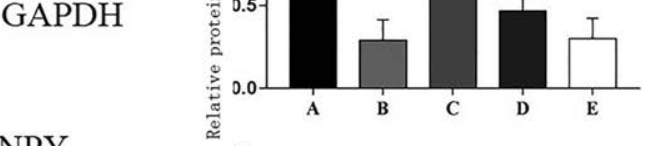
A B
C

E

NPY
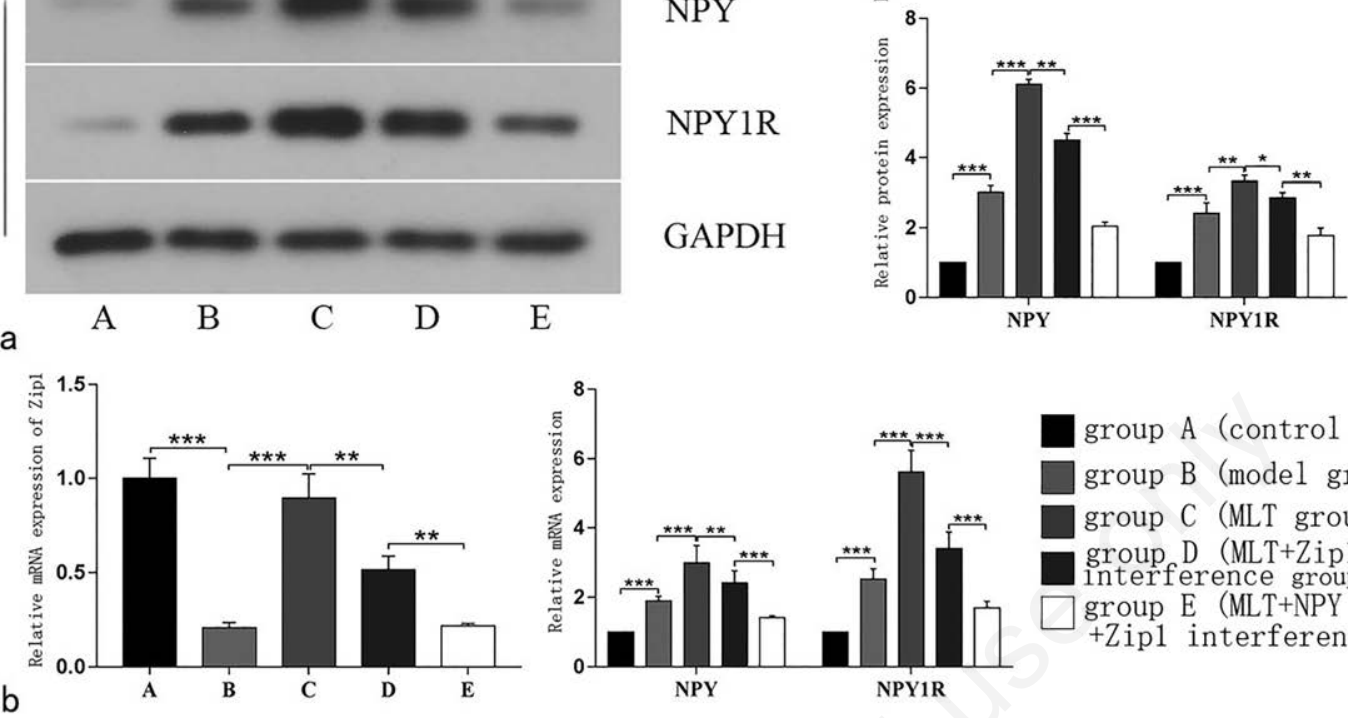

group A (control group) group B (model group) group C (MLT group) group D (MLT+Zip1 interference group) group E (MLT+NPY inhibitor + Zip1 interference group)

Figure 4. Rat model of femoral shaft fracture in each group ( $n=6)$ treated with $1 \%$ alcohol+NS (model group), MLT, MLT+Zip1 interfering lentivirus vector or MLT+BIBP3226+Zip1 interfering lentivirus vector for 8 weeks. a) Western blotting detection of the expression of Zip1, NPY and NPY1R in callus. b) Real-time PCR detection of the expression of Zip1, NPY and NPY1R in callus. Data are shown as mean $\pm S D$ in panels a and $b$. MLT, melatonin; BIBP3226, NPY1R inhibitor; ${ }^{*} p<0.05 ;{ }^{* *} p<0.01 ;{ }^{* * *} p<0.001$. Control groups: $A$ and $B$; $B$ and $C ; C$ and $D ; D$ and $E$.

HE staining $(40 \times)$
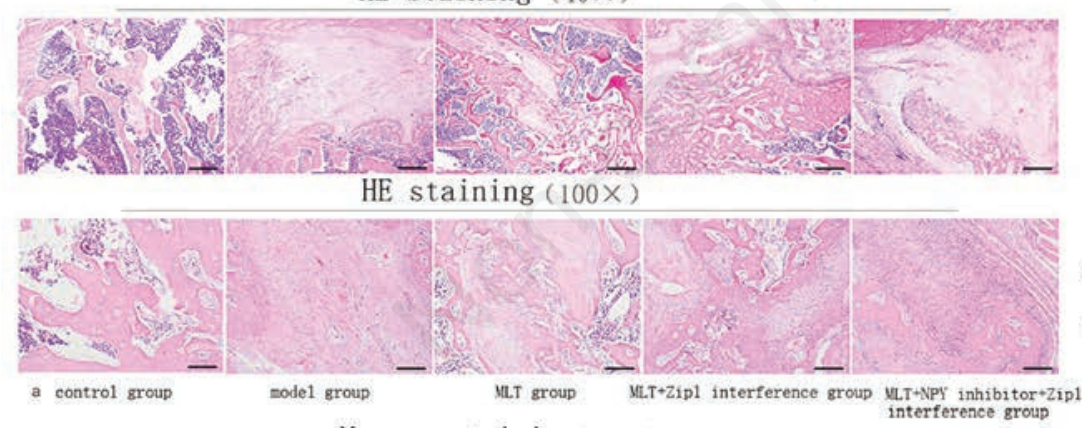

Masson staining $(40 \times)$
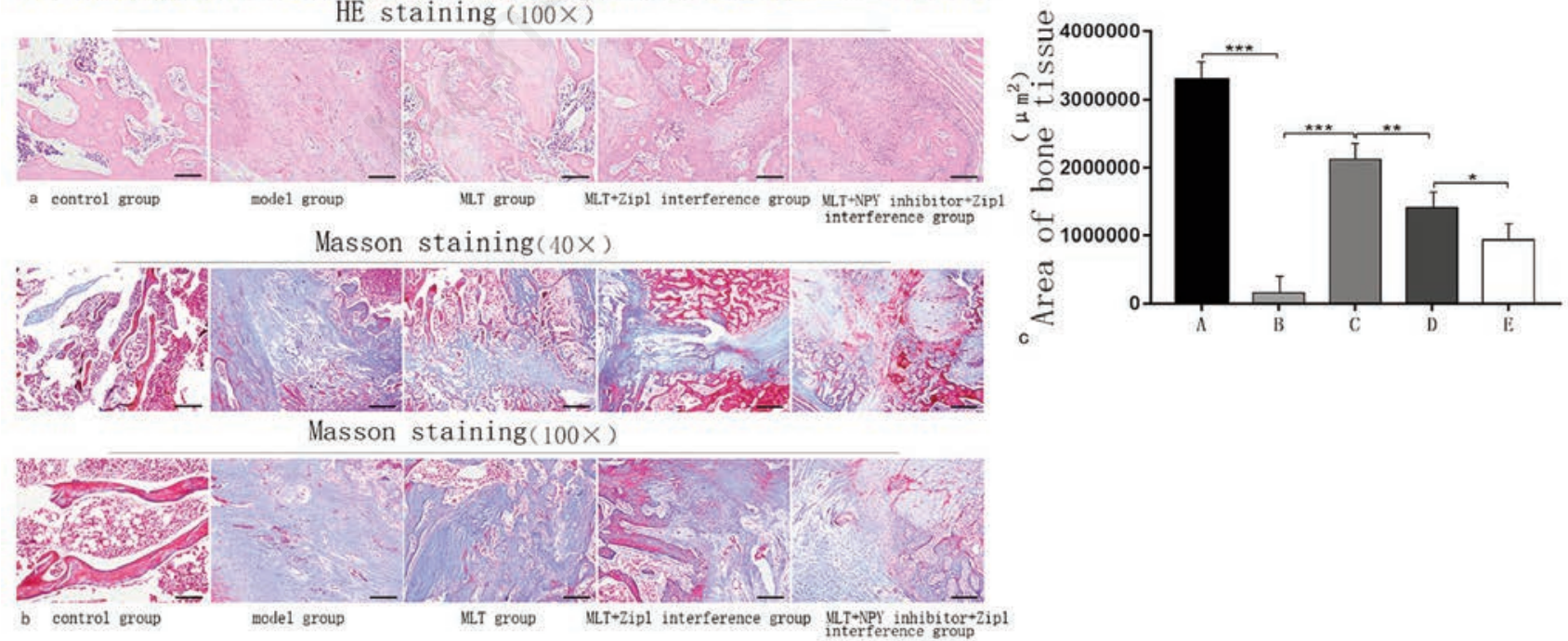

Figure 5. Rat model of femoral shaft fracture in each group $(n=6)$ treated with $1 \%$ alcohol+NS (model group), MLT, MLT+Zip1 interfering lentivirus vector or MLT+BIBP3226+Zip1 interfering lentivirus vector for 8 weeks. a) H\&E staining. b) Masson staining. c) Healing area of bone tissue for the evaluation of osteogenic differentiation. Data are shown as mean \pm SD in panel c. MLT, melatonin; BIBP3226, NPY1R inhibitor; ${ }^{*} \mathrm{p}<0.05 ;{ }^{* *} \mathrm{p}<0.01$; ${ }^{* * *} \mathrm{p}<0.001$. Control groups: A and B; B and C; C and D; D and E. 
Zinc is an essential element that plays an important role in bone growth and development, which has been gradually recognized in recent decades. Balanced zinc nutrition promotes the healthy development of bone, and zinc deficiency may inhibit the growth of bone and reduce bone density. ${ }^{30,31}$ Therefore, the homeostasis of zinc metabolism is of great significance in the process of fracture repair. Our previous study revealed that MLT treatment increased NPY levels. ${ }^{8}$ MLT exerts a protective role in N2a cells via the upregulation of the zinc transporter Zip1 under hypoxia. Zip1 functions as the major endogenous zinc uptake transporter, which transports zinc across the cell membrane. ${ }^{7}$ Increased Zip1 expression induced the osteogenic differentiation of MSCs..$^{5}$ The overexpression of Zip1 may mediate the role of MLT in the promotion of the osteogenic differentiation of MSCs via regulation of NPY and NPY1R levels. Our further research will investigate whether Zip1 is also involved in the process of MLT promotion of MSCs proliferation and migration.

The present study suggests that Zip1 participates in MLT regulation of NPY and NPY1R to promote the osteogenic differentiation of MSCs and fracture healing. The findings in our study provide a reference to understand the mechanism of MLT and clarify the relationship between MLT and Zip1 in the promotion of fracture healing. The results provide further evidence for application in clinical settings.

\section{References}

1. Amstrup AK, Sikjaer T, Heickendorff L, Mosekilde L, Rejnmark L.Melatonin improves bone mineral density at the femoral neck in postmenopausal women with osteopenia:a randomized controlled trial. J Pineal Res 2015;59:221-9.

2. Koerner JD, Vives MJ, O'Connor JP, Chirichella P, Breitbart EA, Chaudhary SB, et al. Zinc has insulin-mimetic properties which enhance spinal fusion in a rat model. Spine 2016;16 777-83.

3. Gaither L A, Eide D J. The human ZIP1 transporter mediates zinc uptake in human K562 erythroleukemia cells. J Biol Chem 2001;276:22258-64.

4. Kessels JE, Wessels I, Haase H, Rink L, Uciechowski P. Influence of DNA-methylation on zinc homeostasis in myeloid cells: Regulation of zinc transporters and zinc binding proteins. J Trace Elem Med Biol 2016;37:125-33.

5. Tang Z, Sahu SN, Khadeer MA, Bai G, Franklin RB, Gupta A. Overexpression of the ZIP1 zinc transporter induces an osteogenic phenotype in mesenchymal stem cells. Bone 2006; 38:181-98.

6. Costello LC, Chellaiah MA, Zou J, Reynolds MA, Franklin $\mathrm{RB}$. In vitro BMP2 stimulation of osteoblast citrate production in concert with mineralized bone nodule formation. J Regen Med Tissue Eng 2015;4:2.

7. Liu XW,Zi Y,Liu YE,Zhang YB,Xiang LB,Hou MX.Melatonin exerts protective effect on $\mathrm{N} 2$ a cells under hypoxia conditions through Zip1/ERK pathway. Neurosci Lett 2015;595:74-80.

8. Ohinata K, Takemoto M, Kawanago M, Fushimi S, Shirakawa $\mathrm{H}$, Goto $\mathrm{T}$, et al. Orally administered zinc increases food intake via vagal stimulation in rats. J Nutr 2009;139:611-6.

9. Dong P, Gu X, Zhu G, Li M, Ma B, Zi Y. Melatonin induces osteoblastic differentiation of mesenchymal stem cells and promotes fracture healing in a rat model of femoral fracture via neuropeptide Y/neuropeptide $\mathrm{Y}$ receptor Y1 signaling. Pharmacology 2018;102:272-80.

10. Long Q, Luo Q, Wang K, Bates A, Shetty AK. Mash1-dependent notch signaling pathway regulates GABAergic neuronlike differentiation from bone marrow-derived mesenchymal stem cells. Aging Dis 2017;8:301-13.

11. Gong Yu, Wang H, Xia H. Stable transfection into rat bone marrow mesenchymal stem cells by lentivirus-mediatedNT-3. Mol Med Rep 2015;11:367-73.

12. Halici M, Oner M, Guney A, Canoz O, Narin F, Halici C. Melatonin promotes fracture healing in the rat model. Eklem Hastalik Cerrahisi 2010;21:172-7.

13. Xue D, Zhang W, Chen E, Gao X, Liu L, Ye C, et al. Local delivery of HMGB1 in gelatin sponge scaffolds combined with mesenchymal stem cell sheets to accelerate fracture healing. Oncotarget 2017;8:42098-115.

14. Gao F, Zhang CQ, Chai YM, Li XL. Lentivirus-mediated Wnt10b overexpression enhances fracture healing in a rat atrophic non-union model. Biotechnol Lett 2015;37:733-9.

15. Radio NM, Doctor JS, Witt-Enderby PA. Melatonin enhances alkaline phosphatase activity in differentiating human adult mesenchymal stem cells grown in osteogenic medium via MT2 melatonin receptors and the MEK/ERK (1/2) signaling cascade. J Pineal Res 2006;40:332-42.

16. Calvo-Guirado JL, Gomez-Moreno G, Mate-Sanchez JE, Lopez-Mari L, Delgado-Ruiz R, Romanos GE. New bone formation in bone defects after melatonin and porcine bone grafts: experimental study in rabbits. Clin Oral Implants Res 2015;26:399-406.

17. Sugiyama O, An DS, Kung SP, Feeley BT, Gamradt S, Liu NQ, et al. Lentivirus-mediated gene transfer induces long-term transgene expression of BMP-2 in vitro and new bone formation in vivo. Mol Ther 2005;11:390-8.

18. Xu XL, Tang T, Dai K, Zhu Zh, Guo XE, Yu Ch, et al. Immune response and effect of adenovirus-mediated human BMP-2 gene transfer on the repair of segmental tibial bone defects in goats. Acta Orthop 2005;76:637-46.

19. Lieberman JR, Daluiski A, Stevenson S, Wu L, McAllister P, Lee YP, et al. The effect of regional gene therapy with bone morphogenetic protein-2-producing bone-marrow cells on the repair of segmental femoral defects in rats. J Bone Joint Surg Am 1999;81:905-17.

20. Virk MS, Conduah A, Park SH, Liu N, Sugiyama O, Cuomo A, et al. Influence of short-term adenoviral vector and prolonged lentiviral vector mediated bone morphogenetic protein-2 expression on the quality of bone repair in a rat femoral defect model. Bone 2008;42:921-31.

21. Su JG, Zhu ZY, Wang Y, Xiong F, Zou J.The cyto-megalovirus promoter-driven short hairpin RNA constructs mediate effective RNA interference in zebrafish in vivo. Mar Biotechnol 2008;10:262-9.

22. Zhang W, Zhang X, Ling J, Wei X, Jian Y. Osteo-/odontogenic differentiation of BMP2 and VEGF gene-co-transfected human stem cells from apical papilla. Mol Med Rep 2016;13:3747-54.

23. Lin Z, Wang JS, Lin L, Zhang J, Liu Y, Shuai M, et al. Effects of BMP2 and VEGF165 on the osteogenic differentiation of rat bone marrow-derived mesenchymal stem cells. Exp Ther Med 2014;7:625-9.

24. Wang Y, He T, Liu J, Liu H, Zhou L, Hao W, et al. Synergistic effects of overexpression of BMP2 and TGFbeta3 on osteogenic differentiation of bone marrow mesenchymal stem cells. Mol Med Rep 2016;14:5514-20.

25. Hsu WK, Sugiyama O, Park SH, Conduah A, Feeley BT, Liu NQ, et al. Lentiviral-mediated BMP-2 gene transfer enhances healing of segmental femoral defects in rats. Bone 2007;40:931-8.

26. Naldini L, Blomer U, Gallay P, Ory D, Mulligan R, Gage FH, et al. In vivo gene delivery and stable transduction of nondividing cells by a lentiviral vector. Science 1996;272:263-7. 
27. Schindeler A, McDonald MM, Bokko P, Little DG. Bone remodeling during fracture repair: the cellular picture. Semin Cell Dev Biol 2008;19:459-66.

28. Iwaki A, Jinguishi S, Oda Y, Izumi T, Shida J-I, Tsuneyoshi M, et al. Localization and quantification of proliferating cells during rat fracture repair: detection of proliferating cell nuclear antigen by immunohistochemistry. J Bone Miner Res 1997; 12:96-102.

29. Rundle $\mathrm{CH}$, Miyakoshi N, Kasukawa Y, Chen S, Sheng Matilda H-C, Wergedal JE, et al. In vivo bone formation in fracture repair induced by direct retroviral-based gene therapy with bone morphogenetic protein-4. Bone 2003;32:591-601.

30. Suzuki T, Katsumata S, Matsuzaki H. A short-term zinc-deficient diet decreases bone formation through down-regulated BMP2 in rat bone. Biosci Biotechnol Biochem 2016;80:1433-5.

31. Wang Xinling, Li Guicai, Zhang Hua, Cui Yong. Comparison of bone mineral density and serum trace elements between Uygur young and elderly women in Urumqi. Chinese J Tissue Engin Res 2015;19:5264-8.

Received for publication: 28 September 2020. Accepted for publication: 5 November 2020.

This work is licensed under a Creative Commons Attribution-NonCommercial 4.0 International License (CC BY-NC 4.0).

CCopyright: the Author(s), 2020

Licensee PAGEPress, Italy

European Journal of Histochemistry 2020; 64(s2):3183

doi:10.4081/ejh.2020.3183 